\section{Probing for a point-of-care TB test}

\section{By Kai-Jye Lou, Staff Writer}

Existing tests to screen patients for tuberculosis are impractical in the pointof-care setting as they are either too slow, lack sensitivity or require specialized lab equipment. ${ }^{1,2}$ Researchers at Stanford University and Texas A\&M University have synthesized highly sensitive class A $\beta$-lactamase-specific fluorogenic probes that could potentially address all three shortcomings. ${ }^{3}$ Global BioDiagnostics Corp. has in-licensed the technology.

Currently, there is no rapid and accurate point-of-care diagnostic for TB infection. Sputum smear microscopy is one of the quickest and simplest methods to screen patients but has low sensitivity, which leads to a high false-negative rate. Culture-based techniques are the gold standard but require several weeks before results are available due to the slow growth rate of Mycobacterium tuberculosis.

Culture methods also require lab equipment and facilities to grow the bacteria.

Newer entrants into the TB testing market include nucleic acid-based strategies that are more sensitive than smear microscopy and can provide results within two hours. However, such tests require expensive, specialized lab equipment and thus are hard to deploy in resource-limited settings. Moreover, these tests do not assay bacterial viability, which would be important for assessing treatment response.

A joint research group co-led by Jianghong Rao and Jeffrey Cirillo has been working on fluorogenic probes that could enable rapid and accurate detection of live M. tuberculosis in clinical samples without the need for specialized lab equipment or facilities.

Rao is an associate professor of radiology and chemistry at Stanford, and Cirillo is a professor in the Department of Microbial and Molecular Pathogenesis at the Texas A\&M Health Science Center.

$M$. tuberculosis expresses class A $\beta$-lactamase (blaC), an enzyme whose hydrolyzing activity the researchers sought to exploit as the mode of activation for their fluorogenic probes.

$\beta$-Lactamases (LACTBs) hydrolyze the $\beta$-lactam ring found in some classes of antibiotics, including penicillins and cephalosporins. Bacterial strains that produce the enzyme resist $\beta$-lactam antibiotics.

In 2010, the research group reported a series of cephalosporin-based probes that fluoresce when hydrolyzed by $\beta$-lactamase. ${ }^{4}$ These probes enabled the detection of $M$. tuberculosis with high sensitivity but lacked specificity for blaC. As a result, $\beta$-lactamases from other bacterial strains also caused the probe to emit a fluorescent signal. Moreover, the probes were slowly hydrolyzed by $\beta$-lactamase and required a few hours before the fluorescent signal was readily detectable.
Now, the researchers have synthesized a new series of cephalosporinbased probes with high specificity for blaC and improved kinetics that generate a detectable fluorescent signal within minutes of exposure to the enzyme.

In vitro, probes exposed to blaC showed 100 - to 200 -fold greater fluorescence than in the absence of blaC. The lead probe showed more than 1,000-fold higher selectivity for blaC over a closely related class $\mathrm{A}$ $\beta$-lactamase that is produced in other strains of bacteria.

The lead probe detected $M$. tuberculosis in unprocessed sputum samples containing as few as 10 colony-forming units and reported a positive result within 10 minutes. Assessments were made using a free luminescence mapping software application for smartphones and photos taken with the smartphone camera.

Results were published in Nature Chemistry.

"Our current probes significantly cut the detection time and can be used with unprocessed clinical samples," said corresponding author Rao. "Using blaC as a detection marker is novel since other methods use DNA or serological markers."

"The chemical probes have low manufacturing cost, show high sensitivity and specificity for the TB enzyme and react with the enzyme in a very rapid manner," added Michael Norman, CEO and cofounder of Global BioDiagnostics. "These properties make the probes attractive for the development of a rapid and cost-effective test that could be used in the point-of-care setting to allow doctors to make a diagnosis of TB when they are still in contact with the patient."

"This study offers early proof of concept that an enzyme expressed by $M t b$ could be detected in sputum samples," added Madhukar Pai, associate professor in the Department of Epidemiology, Biostatistics and Occupational Health at McGill University. "If a simple enzyme detection test can work, and can be done without major investments in equipment or laboratory infrastructure, then that could be a big advance towards a point-of-care solution."

"The approach is an interesting idea and different from conventional detection methods as it relies on a probe that gets modified by an enzyme produced by $M t b$," said Deborah Hung, an assistant professor in the Department of Molecular Biology at Massachusetts General Hospital and in the Department of Microbiology and Immunobiology at Harvard Medical School. "Other methods to detect the pathogen have typically relied on compounds that bind to certain components from the bacterium."

\section{Optimizing TB diagnosis}

Global BioDiagnostics president and cofounder Chris Thornton said that in addition to potentially improving the diagnosis of TB in resource-limited settings, the company's point-of-care test also could help optimize the algorithms used to diagnose TB in countries where more advanced tools and facilities are available.

He gave the example of the semiautomated GeneXpert molecular diagnostics system developed by Cepheid Inc., which is used with Cepheid's Xpert MTB/RIF cartridges to detect $M$. tuberculosis DNA in clinical samples and to assess the bacterium's sensitivity to the antibiotic rifampicin within two hours. 
"A majority of the time, even in resource-constrained settings, TB diagnostic tests come out negative, so from a throughput and cost perspective, it would be advantageous to use our test to initially screen patients for TB and then follow up on just the positive results with systems such as GeneXpert to confirm the result and test for things like drug susceptibility," said Thornton.

"We see our technology as being complementary to the GeneXpert system," Norman told SciBX.

Thornton added that Global BioDiagnostics' point-of-care test also could be useful in monitoring treatment progress. "By requiring live bacteria to trip our test, you can get a quick read on the presence of live bacteria in your samples," he said.

He noted that such treatment monitoring would be difficult with nucleic acid- and smear microscopy-based techniques. Culture-based techniques could be used but require a few weeks to report a result.

Cepheid did not respond to requests for comment.

\section{Built to test}

Global BioDiagnostics is running additional proof-of-concept studies of the fluorogenic probes using clinical samples and has started developing its reagent system to be used with the point-of-care diagnostic assay.

The company hopes to start field testing the diagnostic toward the end of 2014 and to launch the system in 2015. Global BioDiagnostics expects its partner, the Foundation for Innovative New Diagnostics (FIND), to run external clinical trials to validate each step of the diagnostic test development process.

Rao noted that such a system could be a small, single-use, portable device containing the probe with which sputum is collected and transferred into the device, and after a few minutes, read by a small reader or imaged with a cell phone camera. He said the research group also is developing strategies to further improve probe performance to aid the design of nextgeneration fluorogenic probes.

Validation is going to be the key step forward.

"There have been many promising biomarkers that have never made it to the clinic, and therefore I would be keen to see if a prototype assay can be developed and validated in rigorous clinical studies," Pai told SciBX. "I would be keen to see if this test will have high specificity in the presence of nontuberculosis mycobacteria. It will also be important to determine if there is sufficient enzyme activity in clinical samples to ensure high sensitivity."

Hung added that it will also be important to determine the falsepositive rate for a test using the probes. She noted that the $M$. tuberculosis burden in unprocessed clinical samples could be very low relative to other $\beta$-lactamase-producing bacteria such as Pneumococcus and Pseudomonas, so even a 1,000-fold increase in selectivity may not be sufficient to prevent other bacteria from activating the probe.
Last March, Global BioDiagnostics signed a co-development agreement with FIND, under which the foundation will provide funding and help with early stage validation studies of the company's point-of-care TB test.

Norman said it is too early to discuss pricing but told SciBX the company is aiming to have the disposables cost well under $\$ 10$ per test with only one test required per patient and to utilize a reader that costs below $\$ 1,000$.

In June, the Bill \& Melinda Gates Foundation, the World Health Organization's international drug purchase facility UNITAID and the U.S. government signed an agreement with Cepheid to fund the buy down of the Xpert MTB/RIF test to reduce the cost of each test cartridge to $\$ 9.98$ for public sector customers in selected countries. The GeneXpert molecular diagnostic system used to process the test cartridges costs at least $\$ 17,000$ and requires about $\$ 450$ in annual calibration.

Norman said Global BioDiagnostics' point-of-care test will not require any specialized lab instrumentation or tools other than a reader, which will facilitate its deployment to resource-limited settings.

Global BioDiagnostics also has received an undisclosed amount of funding from the Texas A\&M University system, Research Valley Funds LLC and the company's own management. Norman declined to disclose how far the current funding will take the company.

Stanford and Texas A\&M have multiple pending patents covering the fluorogenic probes and the use of bacterial $\beta$-lactamases for diagnostic, imaging and therapeutic applications. Global BioDiagnostics has licensed the patents.

Lou, K.-J. SciBX 5(36); doi:10.1038/scibx.2012.946

Published online Sept. 13, 2012

\section{REFERENCES}

1. McNerney, R. \& Daley, P. Nat. Rev. Microbiol. 9, 204-213 (2011)

2. Pinto, L.M. et al. Med. Princ. Pract. 21, 4-13 (2012)

3. Xie, H. et al. Nat. Chem.; published online Sept. 2, 2012; doi:10.1038/nchem.1435

Contact: Jianghong Rao, Stanford University, Stanford, Calif. e-mail: jrao@stanford.edu

4. Kong, Y. et al. Proc. Natl. Acad. Sci. USA 107, 12239-12244 (2010)

COMPANIES AND INSTITUTIONS MENTIONED

Bill \& Melinda Gates Foundation, Seattle, Wash.

Cepheid Inc. (NASDAQ:CPHD), Sunnyvale, Calif.

Foundation for Innovative New Diagnostics, Geneva, Switzerland

Global BioDiagnostics Corp., Temple, Texas

Harvard Medical School, Boston, Mass.

Massachusetts General Hospital, Boston, Mass.

McGill University, Montreal, Quebec, Canada

Research Valley Funds LLC, College Station, Texas

Stanford University, Stanford, Calif.

Texas A\&M Health Science Center, Bryan, Texas

Texas A\&M University, College Station, Texas

World Health Organization, Geneva, Switzerland 